\title{
SÍNDROME DE WOAKES E SUAS MANIFESTAÇÕES AUDIOLÓGICAS: RELATO DE CASO
}

\section{Woake's syndrome and its auditory manifestations: case report}

\author{
Aline Hanazumi ${ }^{(1)}$, Laura Maria Araújo de Carvalho ${ }^{(2)}$, Daniela Gil ${ }^{(3)}$
}

\begin{abstract}
RESUMO
Tema: manifestações audiológicas na síndrome de Woakes. Procedimentos: A.A.R., uma paciente adulta com diagnóstico de Síndrome de Woakes foi submetida a anamnese, audiometria tonal, logoaudiometria, medidas de imitância acústica e reavaliação das próteses auditivas. Além disso, realizouse um levantamento do prontuário da paciente. Resultados: a análise dos resultados audiológicos em três oportunidades indicou: configuração audiométrica plana, sendo que nos dois primeiros registros foi verificada uma perda auditiva do tipo mista, e, em 2006, uma perda auditiva do tipo neurossensorial de grau moderadamente severo, com curva timpanométrica tipo $\mathrm{B}$, ausência de reflexos acústicos bilateralmente e IPRF compatível com o tipo e grau da perda auditiva. Houve piora de aproximadamente 15 a 20dB nos limiares auditivos por via aérea, e, de 15 a 30 dB via óssea, entre 1996 e 2006. Conclusão: a avaliação audiológica revelou perda auditiva bilateral com alteração das medidas de imitância acústica, sendo que o estudo das manifestações clínicas da Síndrome de Woakes permitiu aceitar a coexistência entre a perda auditiva neurossensorial e curva timpanométrica tipo B.
\end{abstract}

DESCRITORES: Audiologia; Audiometria; Perda Auditiva; Testes de Impedância Acústica

\section{INTRODUÇÃO}

A síndrome de Woakes foi descrita pela primeira vez em 1885 por Edward Woakes ${ }^{1}$, sendo caracterizada por uma etmoidite deformante, com alargamento da pirâmide nasal devido à polipose nasal desde a infância. Esta definição foi ampliada, acrescentando-se a aplasia do seio frontal, bronquiectasia e discrinia (produção de muco altamente viscoso). A discrinia seria responsável por todas as características clínicas desta afecção, por levar a sinusites de repetição, com aplasia do seio frontal e

(1) Fonoaudióloga; Especialização em Distúrbios da Comunicação Humana pela Universidade Federal de São Paulo.

(2) Fonoaudióloga do Núcleo Integrado de Atendimento, Pesquisa e Ensino em Audição da Universidade Federal de São Paulo/Escola Paulista de Medicina, UNIFESP, do Centro de Deficiente Auditivo, CDA do Hospital São Paulo e do Centro Auditivo Teuto Brasileiro, São Paulo, SP; Mestranda em Distúrbios da Comunicação Humana pela Universidade Federal de São Paulo.

(3) Fonoaudióloga; Professora Adjunto do Departamento de Fonoaudiologia da Universidade Federal de São Paulo, UNIFESP, São Paulo, SP; Doutora em Distúrbios da Comunicação Humana pela Universidade Federal de São Paulo.

Conflito de interesses: inexistente formação poliposa, devido à estagnação de muco, a qual bloqueia os ósteos sinusais ${ }^{1,2}$. Outros autores ainda ressaltam que a polipose nasal é uma doença multifatorial, frequentemente associada com asma e outras doenças respiratórias, que resulta de processos inflamatórios imunes crônicos dentro dos seios paranasais ${ }^{3,4}$.

Além disso, a Síndrome de Woakes é mais comum em adultos que em crianças com menos de 10 anos, exceto quando associado à fibrose cística 4,5, e, na população em geral, a taxa de prevalência global da polipose nasal é de 1 a $4 \%{ }^{4}$.

A conservação da audição é uma das funções principais de um audiologista. Conservação e reabilitação formam as bases da existência do campo da audiologia ${ }^{6}$.

As manifestações da síndrome associadas às suas manifestações audiológicas e a raridade de sua ocorrência justificam o estudo da Síndrome de Woakes e suas manifestações audiológicas ímpares.

O objetivo deste relato de caso é descrever os aspectos da Síndrome de Woakes que originam implicações auditivas e os achados audiológicos deste caso. 


\section{APRESENTAÇÃO DO CASO}

Este trabalho foi realizado nos Ambulatórios de Audiologia do Departamento de Fonoaudiologia da Universidade Federal de São Paulo.

A.A.R., 45 anos, sexo feminino, com diagnóstico de Síndrome de Woakes há 17 anos, procurou o ambulatório de Otorrinolaringologia do Hospital São Paulo (HSP - UNIFESP) para acompanhamento do seu caso. A mesma foi encaminhada ao ambulatório de audiologia no mesmo hospital para a realização de nova audiometria e, posteriormente, revisão das próteses auditivas. Os procedimentos realizados foram:

- Anamnese e análise do prontuário da paciente;

- Audiometria tonal (via aérea e via óssea);

- Logoaudiometria (SRT e IPRF);

- Medidas de imitância acústica (timpanometria e pesquisa dos reflexos acústicos);

- Acompanhamento e reavaliação das próteses auditivas.

As audiometrias tonais liminares foram realizadas em cabina acústica utilizando o audiômetro da marca MAICO MA-41, calibrado com as normas da ANSI 1969, com os fones supra-aurais TDH-39 e coxim MX-41. Foram obtidos limiares auditivos nas frequências de 250 a $8000 \mathrm{~Hz}$. Os critérios de normalidade para os limiares de audibilidade neste caso seguiram a classificação de Davis, Silvermann (1970) 7 . As configurações audiométricas foram classificadas de acordo com os critérios da categoria principal, estabelecidos por Lloyde, Kaplan (1978) ${ }^{8}$, para a classificação de curvas descendentes.

A logoaudiometria inclui o Limiar de Reconhecimento de Fala (L.R.F.) e Índice de Reconhecimento de Fala (I.R.F.) ${ }^{9}$. O Limiar de Reconhecimento de Fala consiste na apresentação de palavras dissílabas até ser obtido $50 \%$ de acertos dos estímulos verbais (palavras) que são apresentados em cada orelha. No Índice de Reconhecimento de Fala é apresentada uma lista de 25 palavras monossílabas em cada orelha, sendo os resultados marcados em porcentagem de acertos das palavras (0 a 100\%).

As medidas de imitância acústica foram realizadas utilizando o imitanciômetro da marca AZ7, no qual foram obtidos os valores de compliância, volume da orelha externa (MAE) e pico de máxima compliância. Os volumes do MAE encontram-se na faixa de 0,65 a $1,75 \mathrm{~cm}^{3}$ em adultos ${ }^{10}$. Os resultados da timpanometria foram analisados de acordo com os critérios sugeridos por Jerger (1970) ${ }^{11}$.

Ao analisar o prontuário (com registros a partir de 1989) e anamnese em 2006, verificou-se que a queixa principal da paciente era dificuldade em respirar pelo nariz desde os dez anos de idade. Referiu que a dificuldade para respirar era acompanhada de coriza, espirros frequentes e supuração nasal à esquerda. Além disso, relatou sinusites de repetição, rinite alérgica e bronquite.

De acordo com os dados do prontuário, a paciente foi submetida à cirurgia em 1984, tendo sido realizada uma polipectomia endonasal. No mesmo ano, submeteu-se à outra cirurgia rinológica (técnica de Caldwell-Luc bilateral). Em 1985, foi submetida a uma cirurgia na orelha esquerda, muito provavelmente uma timpanoplastia.

Quatro anos depois, em 1988, foi submetida a uma sinusectomia maxilar bilateral; entretanto, após esta cirurgia houve persistência do quadro respiratório.

A Síndrome de Woakes foi diagnosticada no ano de 1990, mas somente em 1998, foi realizada a colocação de tubo de ventilação em ambos os ouvidos.

A paciente negou antecedentes familiares de deficiência auditiva. Com relação aos antecedentes pessoais, negou diabetes, hipertensão arterial, tuberculose, etilismo ou tabagismo. Referiu ter cefaléia frontal constante, com melhora discreta com o uso de analgésico.

A paciente passou pela primeira vez pelo processo de seleção e adaptação de Próteses Auditivas em Abril de 1999 tendo recebido, por doação, em novembro do mesmo ano, duas próteses auditivas retroauriculares, modelo 215 UT, da marca Danavox. Após esta data a paciente só retornou em Novembro de 2006 para acompanhamento. Foi sugerida a troca dos aparelhos para melhor atender às necessidades da paciente. Foram então realizados novos testes e a nova indicação: duas próteses auditivas retroauriculares, modelo Phoenix 103 da marca Siemens, os quais foram recebidos pela paciente, no início de 2008.

Este estudo contou com a análise e aprovação do Comitê de Ética em Pesquisa da Universidade Federal de São Paulo, sob o protocolo número 1449/07.

\section{RESULTADOS}

A análise dos resultados audiológicos em três oportunidades: Março/1996, Maio/1997 e Abril/2006 indicaram: perda auditiva mista (1996/1997) e perda auditiva neurossensorial bilateral (2006) simétrica, de grau moderado a moderadamente severo, com curva timpanométrica do tipo B bilateral, ausência dos reflexos estapedianos contralaterais bilateralmente; bom IPRF (92\% de acertos em ambas as orelhas). Houve piora de 15 a $20 \mathrm{~dB}$ dos limiares auditivos por via aérea e de 15 a $30 \mathrm{~dB}$ por via óssea, entre 1996 e 2006 (Figura 1). 


\begin{tabular}{|c|c|c|c|c|c|c|c|c|c|c|}
\hline Ano & Orelha & $\begin{array}{c}\text { Frequência } \\
\text { VA/VO }\end{array}$ & 250 & 500 & 1000 & 2000 & 3000 & 4000 & 6000 & $8000(\mathrm{~Hz})$ \\
\hline \multirow{4}{*}{1996} & \multirow{2}{*}{ OD } & VA & 40 & 40 & 45 & 40 & 40 & 35 & 50 & $50(\mathrm{~dB})$ \\
\hline & & VO & - & 25 & 25 & 40 & 40 & $35(\mathrm{~dB})$ & - & - \\
\hline & \multirow{2}{*}{ OE } & VA & 45 & 45 & 50 & 45 & 40 & 35 & 55 & $70(\mathrm{~dB})$ \\
\hline & & VO & - & 30 & 35 & 45 & 40 & $35(\mathrm{~dB})$ & - & - \\
\hline \multirow{4}{*}{1997} & \multirow{2}{*}{ OD } & VA & 55 & 55 & 65 & 55 & 50 & 50 & 50 & $60(\mathrm{~dB})$ \\
\hline & & VO & - & 35 & 35 & 45 & 35 & $45(\mathrm{~dB})$ & - & - \\
\hline & \multirow{2}{*}{ OE } & VA & 60 & 55 & 65 & 55 & 45 & 50 & 50 & $60(\mathrm{~dB})$ \\
\hline & & VO & - & 40 & 45 & 50 & 40 & $50(\mathrm{~dB})$ & - & - \\
\hline \multirow{4}{*}{2006} & \multirow{2}{*}{ OD } & VA & 60 & 55 & 60 & 60 & 55 & 50 & 60 & $55(\mathrm{~dB})$ \\
\hline & & VO & - & 55 & 60 & 60 & 55 & $50(\mathrm{~dB})$ & - & - \\
\hline & \multirow{2}{*}{ OE } & VA & 65 & 60 & 65 & 60 & 55 & 55 & 65 & $60(\mathrm{~dB})$ \\
\hline & & VO & - & 55 & 60 & 60 & 50 & $50(\mathrm{~dB})$ & - & - \\
\hline
\end{tabular}

Legenda:

OD: orelha direita

$\mathrm{OE}$ : orelha esquerda

VA: via aérea

VO: via óssea

$\mathrm{Hz}$ : Hertz

Limiares de VA e VO em dB: decibels

Figura 1 - Limiares de via aérea e via óssea (1996, 1997 e 2006)

\section{DISCUSSÃO}

A Síndrome de Woakes, embora rara ${ }^{4}$, foi descrita há mais de um século ${ }^{1}$. Poucos são os artigos encontrados na Literatura a respeito desta doença e, após uma aprofundada pesquisa nas bases de dados, não foram encontrados artigos sobre a Síndrome especialmente nos últimos cinco anos. Além disso, na Literatura pesquisada, não foi encontrado nenhum estudo que relacionasse as manifestações desta Síndrome aos seus achados audiológicos.

A patogenia da doença ainda é desconhecida. Existem autores que acreditam que há relação com fatores genéticos ${ }^{3}$, enquanto outros não verificaram esta possibilidade em seus estudos $5,13,14$. No caso estudado, embora a paciente tenha negado antecedentes familiares, não houve registro, no prontuário, de pesquisa realizada na genética.

Ao levantar os dados do prontuário da paciente, verificou-se que o início da queixa que caracteriza a Síndrome de Woakes ${ }^{1-4}$ - dificuldade respiratória - ocorreu aos dez anos, idade em que raramente encontram-se casos publicados, embora não tenha havido o registro de uma fibrose cística concomitante, o que é bastante característico da síndrome nesta faixa etária ${ }^{4,5}$. Na anamnese, a paciente referiu que desde criança apresentou dificuldade para respirar pelo nariz, com coriza e espirros frequentes. Apresentou sinusites de repetição, rinite alérgica e bronquite. Além de histórico de otites de repetição, confirmado pelas medidas de imitância acústica (curvas timpanométricas tipo $B$ e ausência de reflexos bilateral) ${ }^{10-12}$. Entretanto, embora as três audiometrias tenham revelado uma configuração audiométrica plana com resultados semeIhantes, nos dois primeiros registros, foi verificada uma perda auditiva do tipo mista e, em 2006, uma perda auditiva do tipo neurossensorial, resultado este, teoricamente incompatível com as medidas de imitância acústica apresentadas pela paciente ${ }^{7}$, as quais por indicarem comprometimento condutivo estariam compatíveis com a presença de gap aéreo ósseo à audiometria tonal por via aérea.

No entanto, tais achados são pertinentes quando se considera o quadro clínico e as manifestações encontradas nesta síndrome.

\section{CONCLUSÃO}

A partir da avaliação audiológica básica e do estudo das manifestações clínicas da síndrome de Woakes conclui-se que a paciente apresenta perda auditiva neurossensorial bilateral de grau moderadamente severo, com configuração audiométrica plana, logoaudiometria compatível com o grau da deficiência auditiva e medidas de imitância acústica, indicando comprometimento de orelha média bilateral.

A partir do estudo das manifestações clínicas da Síndrome de Woakes, pode-se aceitar a coexistência entre a perda auditiva neurossensorial e curva timpanométrica tipo $B$. 


\begin{abstract}
Background: audiological manifestations in Woake's Syndrome. Procedure: a female adult patient diagnosed with Woake's Syndrome has undergone clinical history, pure tone audiometry, speech audiometry, acoustic immittance measures and revision of hearing aids. Her record files were also reviewed. Results: three different pure tone audiometries were compared and indicated: flat audiometric configuration, with two evaluations indicating mixed hearing loss, and, in 2006, a moderate-to-severe sensorineural hearing loss with flat tympanograms, absence of acoustic reflexes bilaterally and good WRS. An increase of about $15 \mathrm{~dB}$ to $20 \mathrm{~dB}$ of pure tone thresholds and $15 \mathrm{~dB}$ to $30 \mathrm{~dB}$ of bone thresholds was observed in 10 years of evolution. Conclusion: the study of the clinical manifestations concerning Woakes's Syndrome allowed the acceptance of the coexistence between the sensory neural hearing loss and the type B tympanometric curve.
\end{abstract}

KEYWORDS: Audiology; Audiometry; Hearing Loss; Acoustic Impedance Tests

\section{REFERÊNCIAS}

1. Woakes E. Necrotising ethmoiditis and mucous polyps. Lancet. 1885; 61:619.

2. Kellerhals B, Uthemann B. Woakes' Syndrome: the problems of infantile nasal polyps. Int J Pediatr Otorhinolayngol. 1979; 1:79-85.

3. Caversaccio M, Baumann A, Helbling A. Woakes' Syndrome and albinism. Auris Nasus Larynx. 2006; 34(2):245-8.

4. Pawankar R. Nasal polyposis: an update: editorial review. Curr Opin Allergy Clin Immunol. 2003 Feb; 3(1):1-6.

5. Groman JD, Bolger W, Brass-Ernst L, Macek Junior MD, Zeitlin P, Cutting G. Recurrent and destructive nasal polyposis in 2 siblings: a possible case of Woakes' Syndrome. Otolaryngol Head Neck Surg. 2004; 131(6):1009-11.

6. Melnick W. Saúde auditiva do trabalhador. In: Katz J. Tratado de audiologia clínica. 4. ed. São Paulo: Manole; 1999. p.529-45.

7. Davis H, Silverman SR. Hearing and deafness. 3. ed. New York: Holt, Rinehart \& Winston; 1970.
8. Lloyd LL, Kaplan H. Audiometric interpretation: a manual of basic audiometric. Baltimore: University Park Press; 1978.

9. Pen MG, Mangabeira-Albernaz PL. Desenvolvimento de testes para logoaudiometria: discriminação vocal. Anales do Congresso Pan-Americano de Otorrinolaringologia y Broncoesofasologia, Lima, Peru. 1973. vol. 2, p. 223-6.

10. Holte L, Margolis $\mathrm{RH}$, Cavanaugh Junior RM. Developmental changes in multifrequency tympanograms. Audiology. 1991; 30(1):1-24.

11. Jerger JF. Clinical experience with impedance audiometry. Arch Otolaryngol. 1970; 92(4):311-24.

12. Fria T, LeBlanc J, Kristensen R, Alberti PW. Ipsilateral acoustic reflex stimulation in normal and sensorineural impaired ears: a preliminary report. Can J Otolaryngol. 1975; 4(4):695-703.

13. Canuyt M, Terracol J. La polypose nasal récidivante et déformante des jeunes. Rev Laryng Otol. 1924; 45:7-10.

14. Augusto AGLBS, Granato L, Santos GC, Carvalho MFP. Síndrome de Woakes: a propósito de dois casos. Rev Bras Otorrinolaringol. 1998; 64(1):81-6.

RECEBIDO EM: 27/11/2008

ACEITO EM: 09/04/2009

Endereço para correspondência:

Aline Hanazumi

Rua Epaminondas de Oliveira, 174

São Roque - SP

CEP: 18130-505

E-mail: hanazumi@gmail.com 